\title{
L'Attention
}

\section{Attention suscitée et dirigée}

Bernhard Waldenfels

\section{OpenEdition}

Journals

Édition électronique

URL : http://journals.openedition.org/alter/1527

DOI : $10.4000 /$ alter. 1527

ISSN : 2558-7927

\section{Éditeur :}

Association ALTER, Archives Husserl (CNRS-UMR 8547)

\section{Édition imprimée}

Date de publication : 1 octobre 2010

Pagination : 33-44

ISBN : 2-9522374-6-8

ISSN : 1249-8947

Référence électronique

Bernhard Waldenfels, «Attention suscitée et dirigée », Alter [En ligne], 18 | 2010, mis en ligne le 01 juin 2020, consulté le 28 juin 2020. URL : http://journals.openedition.org/alter/1527 ; DOI : https://doi.org/ 10.4000/alter.1527 


\title{
ATTENTION SUSCITÉE ET DIRIGÉE
}

\author{
Bernhard Waldenfels
}

Il est évident que l'attention ne fait pas partie des grands thèmes de la philosophie occidentale en tant que celle-ci s'occupe de l'être, du temps, de l'espace, de la liberté ou du sujet. Une telle réserve donne à penser. Il me semble qu'elle s'explique par quelques points faibles, inhérents aux interprétations traditionnelles. Premièrement, l'attention se réduit à une étape préparatoire, à une double fonction : il faut ouvrir les yeux et dresser les oreilles, et le reste viendra. Deuxièmement, l'attention s'appuie sur d'autres éléments, par exemple sur les intentions de la volonté, sur les tentatives de mettre quelque chose à jour ou sur les états de sommeil et de veille. Troisièmement, l'attention tend à être scindée en deux, particulièrement en la dualité des actes et des mécanismes ou de l'activité et de la passivité, et cela en correspondance avec le dualisme cartésien. Quatrièmement, il manque d'une question directrice centrale. L'idée de l'attention a donc des traits nomadiques et bâtards qui l'empêchent d'occuper une position forte.

Il est vrai que dans le cours de la pensée occidentale, nous rencontrons des philosophes comme Plotin, Saint Augustin, Descartes, Locke, Malebranche ou Leibniz qui concèdent à l'attention une certaine place dans la vie de l'esprit, de la conscience ou de l'action. Pourtant il faut attendre longtemps pour que l'attention compte réellement. La deuxième moitié du XIXe siècle est fortement dominée par l'éclecticisme d'un Wilhelm Wundt qui dégradait l'attention en une fonction purement psychologique. C'est seulement au seuil du XXe siècle que trois grandes figurent apparaissent: William James, Henri Bergson et Edmund Husserl, qui proposent une nouvelle manière de radicaliser l'expérience, bien loin des perspectives également insuffisantes de l'empirisme et du psychologisme. Chacun d'eux cherchait à sa façon à placer l'attention au cœur de l'expérience. Ces tentatives étaient accompagnées par des efforts similaires en provenance de divers domaines, comme la théorie et la technologie de la perception, l'organisation du travail ou encore le champ des beaux-arts, en particulier de la peinture, de la musique et de la photographie. Je propose ici de mettre en relief quelques motifs fondamentaux 


\section{L'attention}

d'une théorie de l'attention, basés sur certaines présuppositions de ce que j'appelle une phénoménologie responsive ${ }^{1}$.

\section{L'attention comme fait originaire}

Le rôle décisif que nous attribuons à l'attention dépend de quelques présuppositions qui peuvent paraître fort modestes. En réalité, elles ne sont pas si simples. Au fond, c'est le fait que quelque chose apparaît, que ceci apparaît plutôt qu'autre chose et qu'il apparaît de cette manière plutôt que d'une autre. En ce point crucial, nous tombons sur un fait originaire que Goethe aussi bien que Husserl et Wittgenstein appellent l'Urphänomen. Celui-ci ne constitue point un principe dont on peut dériver quelques propositions. Il est originel en tant que le point de départ à partir duquel on pose les questions quasi officielles de la philosophie telles que : Qu'est-ce que c'est? Qui suis-je? Qui es-tu ? Pourquoi se passe-t-il quelque chose? Les faits originaires suscitent de telles questions avant que nous soyons capables de décrire et de définir ce qui se passe. Prenons un bruit soudain dans la rue, une explosion, un coup de foudre, un accident de voiture, une douleur piquante; ou prenons un phénomène céleste anormal, une idée inattendue qui vient, un Einfall (à savoir une sorte d'invasion), ou prenons l'inspiration liée au souffle de la respiration. Quelle que soit la chose qui suscite notre attention, $c^{\prime}$ est seulement après coup que se pose la question de savoir ce qu'est ce qui nous a effrayés, étonnés ou surpris d'une manière extraordinaire.

Avant de déployer cette idée, j'insère une brève remarque méthodique et un commentaire historique. Le fait originel est quelque chose qu'on ne peut que montrer, c'est-à-dire indiquer d'une manière indirecte, postérieure sans être capable de le démontrer. Ainsi en allemand nous distinguons entre aufweisen et beweisen. Néanmoins, nous sommes forcés de montrer que ce fait étrange est réellement originaire et primaire, sous peine d'en venir à des affirmations dogmatiques entraînant un certain fondamentalisme ${ }^{2}$. C'est à Aristote qu'il revient d'avoir remarqué que dans le champ du raisonnement pratique, le «que» (hoti) précède le «parce que»(dihoti) $)^{3}$. Si bien que la possibilité de décider quel argument joue et quel principe doit être appliqué dépend de la définition de la situation. En outre, il faut prendre en

1. Concernant les dimensions diverses de l'attention cf. livre de l'auteur Phänomenologie der Aufmerksamkeit, Frankfurt/M., Suhrkamp, 2004. Le cadre théorétique de la phénoménologie responsive est développé en détail dans Antwortregister, Frankfurt/M., Suhrkamp, 1994, et dans une forme plus serrée dans Grundmotive einer Phänomenologie der Erfahrung, Frankfurt/M., Suhrkamp, 2006, partiellement traduit en français par F. Gregorio, F. Moinat, A. Renken et M. Vanni dans : Revue de Théologie et de Philosophie, vol. 137 (2005/IV), p. 291-426. Une Philosophie de la réponse, Bernhard Waldenfels, Choix de textes.

2. Cf. de l'auteur « Description indirecte », Archives de Philosophie 73 (2010), p. 29-45.

3. Aristote, Éthique à Nicomaque, I, 1. 
considération le rôle de la chance (tyché), qui peut contrecarrer nos desseins. Même Kant, fermement décidé à légitimer nos jugements et nos actions, recourt à une démonstration ostensive dans laquelle la conviction de la vérité est liée à la vue dans leurs sources ${ }^{4}$.

\section{L'attention comme événement double et intermédiaire}

Une description minimale, semblable à ce que les représentants du «Minimal Art » produisent par le moyen des couleurs et des lignes, révèle que l'attention est un événement double : quelque chose me frappe-moi, je fais attention. Dans ce contexte je préfère user d'expressions verbales plutôt que nominales parce qu'elles sont plus proches de la genèse de l'expérience ; ainsi, en allemand : etwas fällt mir auf-ich merke auf. L'un des deux membres de phases correspond à l'attention suscitée, l'autre à l'attention dirigée. Cette description, d'apparence assez simple, doit être développée ; elle inclut en effet une série d'aspects essentiels.

(1) La première partie de l'attention est constituée par le fait que quelque chose $m^{\prime}$ arrive, me frappe, me touche, m'affecte. Pour le caractériser je fais usage du mot allemand Widerfahrnis, qui littéralement signifie une espèce de contre-expérience, ou peut renvoyer au mot grec pathos, considéré dans son triple sens: la forme grammaticale du passif, la souffrance et la passion. Nous avons affaire ici à un événement, ou plutôt à un avènement : nous y sommes engagés, non pas au nominatif de l'auteur, mais plutôt au datif ou à l'accusatif, c'est-à-dire sous des formes grammaticales qui se réfèrent à moi comme à quelqu'un qui est concerné ou à qui on s'adresse. Pour reprendre la proposition de Karl Bühler, on pourrait parler d'un "datif de l'adresse », à savoir d'une forme dative utilisée pour s'adresser à quelqu'un ${ }^{5}$.

(2) La deuxième partie de l'événement double est à entendre comme une réponse que je donne ou refuse, au-delà de son contenu propositionnel. En disant ou faisant quelque chose, l'événement de la réponse ne coïncide pas avec le dit et le fait. Le verbe "répondre " se réfère à un événement qui ne fait purement qu'arriver, mais cet événement se transforme en un acte de langage (speech act) or en une action pratique qui, de leur côté, reçoivent un certain sens et suivent certaines règles. Prenons une séquence des plus ordinaires. Quelqu'un me demande : "Quelle heure est-il ? », et je réponds : «Trois heures. » En donnant cette réponse je remplis une certaine lacune de savoir ; mais simultanément je réponds à la demande de l'autre. Cela paraît assez simple. La situation serait déjà moins triviale si quelque chose d'important était en jeu, c'est-à-dire si ce qui est en jeu n'était pas déjà pour ainsi dire consigné d'avance dans le livre de l'histoire, mais y ouvrait une page nouvelle. Il faut considérer ici des événements singuliers comme la

4. I. Kant, Critique de la Raison pure, B 817.

5. K. Bühler, Sprachtheorie, Stuttgart, G. Fischer, 1982, p. 251. 


\section{L'attention}

naissance d'un individu ou le déclenchement d'une guerre ou d'une révolution.

(3) L'événement de l'attention se présente comme un événement double et intermédiaire, franchissant un seuil qui tout à la fois joint et sépare, comparable en cela au trait d'union ${ }^{6}$. D'un côté, il joint. Car il n'y a pas de pathos sans quelqu'un à qui il arrive, qu'il s'agisse d'un événement qui fait plaisir et réjouit, ou qui provoque de la douleur et donne du souci. Inversement, il n'y a pas de réponse sans quelque chose ou quelqu'un à qui elle répond. De l'autre côté, le seuil sépare ; car aucun des deux aspects ne peut être dérivé de l'autre. Nous ne nous trouvons pas seulement en face d'une différence de qualité, mais d'une différence de direction, étant donné que quelque chose vient à moi ou bien part de moi. Les deux mouvements ne peuvent pas être intégrés dans un tout; par conséquent ils résistent à la totalisation ou à la réconciliation au sens hégélien.

(4) Le fait que quelque chose nous arrive exclut deux extrêmes. D'une part, il exclut toute sorte de fondamentalisme, présumant que la réponse serait donnée dans les choses. Les réponses doivent être inventées et ne sont pas disponibles là comme des choses toutes faites qu'on pourrait se contenter de ramasser. Cependant, ce qui se trouve également exclu, c'est toute sorte de constructivisme pour lequel il $\mathrm{n}^{\prime} \mathrm{y}$ aurait rien à quoi l'on répond mais seulement des matériaux dont on fait quelque chose. Les constructivismes de toute sorte supposent une sorte de fabrication qui perd de vue ses propres présuppositions et motivations.

(5) L'hiatus entre le pathos et la réponse, étant franchi par l'attention sans être aboli, s'accompagne d'une scission du soi-disant sujet. Le sujet se divise en deux figures : d'une part le patient (au sens large du mot), d'autre part le répondant. Nous devenons ce que nous sommes, en étant affectés et en y répondant. Donc nous ne serons jamais complètement ce que nous sommes. Ainsi Paul Valéry écrit-il : «Ce que JE suis instruit, étonne ce que je suis. Et il y a un temps entre moi et moi. Moi naît de moi ${ }^{7}$. En ce point crucial, la phénoménologie responsive croise la psychanalyse de Freud et de ses successeurs, comme Lacan et Laplanche. A supposer que notre expérience commence par ce qui nous arrive en tant que patient, nous serons jamais le maître de nous-même. En conséquence, des para-phénomènes comme l'inconscience et l'in-volontaire ne sont pas à définir par un simple manque de connaissance et de volonté, mais par une expérience originelle de retrait, d'absence - bref, par une expérience spécifique de l'étranger. La force de notre créativité s'avère le revers d'une faiblesse originelle et interne ${ }^{8}$.

6. Cf. le terme anglais hyphen qui est dérivé du grec et signifie littéralement «au-dessous de l'un ", suggérant une sorte d'unification insolite.

7. P. Valéry, Cahiers, vol. I, Paris, Gallimard 1973, p. 1001.

8. C'est Maurice Merleau-Ponty qui se réfère à une "faiblesse interne qui nous empêche d'obtenir jamais la densité d'un individu absolu»; cf. Phénoménologie de la perception, Paris, Gallimard, 1945, p. 489. Concernant l'échange fructueux entre phénoménologie et psychanalyse, qui a une longue histoire, particulièrement en France, je renvoie à mes propres analyses 
En résumé, l'événement de l'attention ne se réduit ni à un collage de mécanismes extérieurs et d'actes intérieurs, ni à une échelle conduisant continûment de la passivité à l'activité. Au contraire, cet événement étrange est pénétré par une passivité radicale et originaire. Introduite par Husserl et radicalisée par Levinas, une telle passivité signifie plus que le contraire de notre propre activité et plus qu'un degré inférieur d'activité. Répondre veut dire commencer ailleurs, commencer par quelque chose qui se soustrait. En répondant à la demande de l'autre, nous sortons de nous-même.

\section{L'attention comme déplacement temporel}

L'événement double de l'attention ouvre un chapitre nouveau dans le grand livre du temps. Ce chapitre se qualifie par une temporalité particulière. Nous avons mentionné les deux mouvements que sont l'être affecté et le répondre. Ceux-ci sont séparés par un déplacement temporel, par une sorte de time-lag que j'appelle diastase. Ce terme ancien, introduit par Aristote et Plotin et repris par Levinas, signifie littéralement « écartement de soi ». La diastase prend un sens spatio-temporel. Pendant que nous nous trouvons ici et maintenant nous sommes aussi bien ailleurs, et cela d'une manière paradoxale qui contredit les conceptions communes. Tout ce qui nous arrive, nous frappe, nous touche et nous surprend vient trop tôt, tandis que la réponse que nous y donnons vient trop tard. Il s'agit d'une antériorité et postériorité tout originaires. Bien entendu, venir trop tôt ou venir trop tard ne veut pas dire que l'un vient plus tôt que l'autre, comme s'il s'agissait d'événements placés sur des points successifs d'une échelle continue du temps. Une telle succession correspond à la perspective de l'observateur et non point à celle du patient. Vu sous l'angle de l'expérience même, nous sommes tout à la fois plus jeune et plus vieux que nous-mêmes, comme le dit Platon à l'égard de ce qui se passe dans le temps ${ }^{9}$.

\section{Attention et sélection}

La sélectivité de l'attention est un motif assez commun, mais il convient de le préciser à la lumière de l'expérience. Évidemment, il n'y a aucune attention sans sélection : se tourner vers ceci veut dire aussi se détourner de cela. Ce va-et-vient, rendu en allemand par Zuwendung et Abwendung,

dans Bruchlinien der Erfahrung. Phänomenologie, Psychoanalyse, Phänomenotechnik, Frankfurt/M., Suhrkamp, 2002.

9. Cf. Platon, Parménide 141 c-d. Husserl s'approche de cette passivité élémentaire du temps dans ses leçons de Göttingen de 1904-1905, partiellement publiées sous le titre Wahrnehmung und Aufmerksamkeit. Texte aus dem Nachlaß (1893-1912) (Hua XXXVIII), Dordrecht, Springer, 2004 (trad. fr. par N. Depraz, Phénoménologie de l'attention, Paris, Vrin, 2009. Mais il me paraît que Husserl ne va pas suffisamment loin. Voir mon compte-rendu «Wahrnehmung und Aufmerksamkeit beim frühen Husserl », Philosophische Rundschau 52 (2005), p. 302-310. 


\section{L'attention}

correspond au double «plutôt », contenu dans notre formule initiale, disant que ceci apparaît plutôt qu'autre chose et qu'il apparaît de cette manière plutôt que d'une autre. Cette préférence élémentaire nous renvoie aux formes privilégiées de la Gestalt-Theorie; elle exclut l'hypothèse d'un panorama qui nous laisserait voir toutes les choses à la fois. En effet, on voit toujours plus qu'on ne regarde, et on entend plus qu'on n'écoute. Un tel processus de sélection, placé au cœur de notre attention, résiste à certaines interprétations simplificatrices. D'un côté, il résiste à une interprétation réaliste selon laquelle l'attention se contente de choisir quelque chose qui serait déjà donné. C'est ainsi que David Hume présume que, dirigés par notre imagination, nous ne faisons rien d'autre que trier (pick out) les idées les plus appropriées à notre but ${ }^{10}$. Même William James définit l'attention comme "la sélection de quelque chose et la suppression du reste ", assez proche de la façon dont le sculpteur travaille un bloc de marbre ${ }^{11}$. Ce préjugé se prolonge jusque dans la comparaison de l'attention à la lumière d'un projecteur, fortement critiquée par Aron Gurwitsch et par Merleau-Ponty ${ }^{12}$. De l'autre côté, la sélection s'oppose à une interprétation subjectiviste à la manière de Wundt, qui fut vivement attaquée par Husserl. Le célèbre fondateur de la psychologie expérimentale réduit en effet l'attention à un «état, caractérisé par des sentiments particuliers qui accompagnent l'appréhension claire d'un contenu psychologique ${ }^{13}$ ». Cependant, la sélection, liée à l'étroitesse de la conscience et aux horizons limités du sens, n'est pas un phénomène marginal; elle fait partie intégrante de l'organisation de l'expérience même. Tout voir à la fois signifierait ne rien voir.

\section{L'attention comme réponse créative}

Les effets de l'attention ne se limitent pas à la sélection de certains contenus, ils se manifestent dans certains modes pas lesquels les choses sont données (Gegebenheitsweisen) et les actes accomplis (Vollzugsweisen). Ceux modes de l'expérience n'existent ni dans le monde extérieur des choses physiques ni dans le monde intérieur des actes ou des états mentaux : ils sont à inventer ou à créer. Plus précisément, ils se créent par l'organisation du champ de l'expérience et par la détermination de l'indéterminé. Cependant, cette création n'équivaut pas à une création pure qui nous transporterait directement dans un monde imaginaire. Au contraire, la création s'accomplit à travers des réponses qui sont créatives en tant que réponses. Passant par ce processus de création responsive, ce par quoi (Wovon) nous sommes affectés et à quoi

10. D. Hume, A Treatise of Human Nature, I, 1.7.

11. W. James, Principles of Psychology, New York, Dover Publ., 1950, vol. I, p. 288.

12. Voir M. Merleau-Ponty, loc. cit., p. 36-40, et remontant plus loin Aron Gurwitsch, Théorie du champ de la conscience, Bruges, Desclée de Brouwer, 1957; Studies in Phenomenology and Psychology, Evanston, Northwestern, 1966.

13. Cf. W. Wundt, Grundriß der Psychologie, Leipzig, Engelmann, 111913, p. 252. 
(Worauf) nous répondons se transforme en quoi (Was). Il en résulte quelque chose que nous visons comme quelque chose, intégré dans certaines structures et soumis à certaines règles. Dans Sein und Zeit Heidegger fait une distinction similaire entre le Wovor de l'angoisse et le Was de la peur : la peur concerne quelque chose dans le monde, tandis que l'angoisse touche le monde même.

\section{En deçà et au-delà du sens et de la règle}

L'attention créative implique une dimension spéciale de l'expérience, que nous caractérisons comme pathique et responsive. Ce qui se passe dans cette profondeur créatrice précède et excède toute sorte de sens et de règle; cela se tient en deçà et au-delà de l'intentionnalité et de la régularité. Quelle que soit la chose qui nous frappe ou nous affecte, de prime abord elle n'a pas de sens et ne suit aucune règle, mais reçoit du sens et des règles par la créativité de nos réponses. Pour déceler cette dimension profonde, nous avons besoin d'une épochè tout à fait spéciale. Ce qu'il faut, c'est une épochè responsive, ce qui comprend une épochè attentive. Cette dernière nous fait interrompre le cours normal de l'expérience naturelle; mais contrairement à l'épochè commune, elle ne s'arrête pas au sens que notre expérience vise, mais nous mène au-delà de lui vers ce à quoi (ou à qui) nous répondons. En fin de compte, nous touchons à ce qui nous attend sans être capable de l'anticiper.

\section{Attention incorporée et structurée}

Ce qui suit n'est plus qu'une esquisse destinée à marquer certaines charnières. D'abord, l'attention ne se réduit pas à des fulgurations momentanées ; elle s'incorpore dans un ensemble de structures et d'habitudes. Du côté des objets et des buts, nous découvrons des traits frappants (Auffälligkeiten) qui se répètent, produisant un monde d'indices et d'effets qui nous rappellent le Merkwelt et le Wirkwelt de Jakob von Uexküll ${ }^{14}$. Dès que nous passons du côté du comportement subjectif, nous trouvons des habitudes et des dispositions de toutes sortes qui nous prédisposent à être attentifs, constituant une certaine Aufmerksamkeitsbereitschaft ou readiness. Cette double formation de l'attention, mi-structurale, mi-habituelle, joue un rôle spécial dans les mondes professionnels, soutenant par exemple le diagnostic médical ou le flair du détective. Je choisis un exemple, emprunté au contexte du trafic de drogue. Comment se fait-il que l'agent de police, chargé de faire des recherches à l'aéroport, découvre quelle personne porte des drogues sur elle? On raconte l'histoire d'un agent particulièrement habile, sachant diriger son attention vers tout voyageur regardant anxieusement çà et là ; il est à supposer que de telles personnes se signalent

14. Cf. J. Von Uexküll, Theoretische Biologie, Frankfurt/M., Suhrkamp, 1973. Ce livre fondamental, premièrement publié en 1928, était bien connu des premiers phénoménologues. 


\section{L'attention}

comme suggérant qu'elles ont quelque chose à cacher. Elles deviennent voyantes, auffällig. C'est d'une manière analogue que nous parlons des symptômes, par exemple des rougeurs cutanées, déchiffrées par un médecin attentif comme les indices d'une fièvre imminente.

Comme ces exemples le suggèrent, ce sont surtout des phénomènes déviants qui attirent le regard et éveillent l'attention. Cette constitution d'un monde de l'attention a certaines implications et conséquences.

(1) D'abord, il faut distinguer entre une attention primaire, innovatrice, et une attention secondaire, normale. Dans le premier cas notre expérience est ébranlée par quelque chose qui nous surprend, nous étonne ou nous effraie, tandis que dans le deuxième nous voyons ou entendons quelque chose que nous connaissons déjà dans une certaine mesure. L'attention devient quelque chose de banal tant que nous négligeons cette distinction. Par suite, les impératifs de la vie quotidienne comme «Faites attention! » ou simplement «Attention!» semblent ne faire que redoubler l'expérience bien qu'ils fassent toujours plus que cela. Même le comportement qu'on dit machinal n'est jamais complètement mécanique ; il est plus ou moins mécanisé. De là vient que le keep smiling stéréotypé se déforme en le sourire glacé de Marilyn Monroe, représenté par Andy Warhol d'une manière quasi mécanique.

(2) Le processus bilatéral de stabilisation par des structures et des habitudes, qui modèle sans cesse notre attention, engendre une sphère intermédiaire des pratiques, des techniques et des médias. Cette sphère est peuplée par des gardiens, des moniteurs ou des alarmes automatiques, elle inclut des modes de comportement vigilants, et crée de cette manière un style d'attention qui varie d'une culture à l'autre. Par conséquent, l'attention prend des formes variées et contingentes. À la suite de Nietzsche, nous pouvons défendre l'idée que l'être humain, partiellement délivré des chaînes de l'instinct, est en réalité une espèce $d^{\prime}$ " «animal non fixé ». Surchargée de stimuli et d'attraits, l'attention humaine a besoin de certains modes de «fixation » qui font partie de notre culture. La technologie et l'économie de l'attention, y compris la lutte pour l'attention, engendrent une telle quantité de problèmes qu'on ne peut ici que les effleurer ${ }^{15}$.

(3) Finalement, toutes ces attitudes et habitudes sont enracinées dans notre corps qui, en tant que Leibkörper, se présente tout à la fois comme corps vivant (Leib) et comme corps matériel (Körper), y compris le cerveau. La matérialité s'explique par le fait que notre corps fait partie de ce qu'il constitue. Les neurologues ont découvert certains mécanismes d'inhibition réciproque, intervenant dans les processus qui se déroulent dans un seul sens, entre les sens différents ou entre la cognition et l'émotion; ils nous aident à réduire la complexité de notre vie corporelle. Pour explorer en détail l'incorporation de l'attention, la phénoménologie doit être complétée

15. Voir plus en détail ma Phänomenologie der Aufmerksamkeit, loc. cit., ch. V. Quant au contexte culturel et scientifique de l'attention, cf. Jonathan Crary, Suspensions of Perception, Cambridge, MIT Press, 1999 ; mais l'arrière-fond philosophique a besoin d'être approfondi et complété. 
par une espèce de phénoménotechnique. Pour le dire en grec, il n'y a pas de logos sans techne $e^{16}$.

\section{L'attention polarisée et bloquée}

En ce qui concerne les possibilités variables et les anomalies de l'attention, il faut prendre en considération le fait que les parts pathique et responsive de l'attention ne sont que rarement en équilibre. L'attention est soumise à un certain destin qui ressemble au destin des pulsions chez Freud. Ce destin se manifeste par des formes multiples.

D'abord, l'attention se présente comme polarisée. D'une part, nous sommes tentés de pratiquer un certain laisser-faire. Nous laissons vagabonder nos pensées et nos désirs. Avec la rêverie, nous tombons dans un rêve diurne, déjà décrit par Rousseau, Locke et Leibniz; il se déroule dans l'antichambre de l'ego ${ }^{17}$. La flânerie, en tant qu'une promenade sans but et comme forme élégante de Müßiggang, d'oisiveté, anime la vie bourgeoise publique des grandes villes; elle est méticuleusement dépeinte par des auteurs comme Charles Baudelaire, Walter Benjamin ou Franz Hessel. Le brainstorming est pratiqué pour sa part comme un entraînement de la conscience qui nous aide à relâcher le flux des idées et à stimuler l'élaboration des projets. Mais cela n'est que l'un des côtés de la médaille. De l'autre côté, nous suivons le chemin de la concentration, en fixant une certaine tâche et en mettant de côté tout ce qui pourrait nous déranger. En général, notre comportement oscille entre la dispersion et la collection, mais depuis l'époque de Saint Augustin jusqu'à aujourd'hui, notre tradition philosophique et théologique manifeste une prédilection pour la dernière. Quant aux cultures de l'Extrême-Orient, certaines pratiques corporelles comme le tir à l'arc et certains exercices spirituels comme la méditation zen constituent des écoles de l'attention toutes particulières. Quoi qu'il en soit, portées à l'extrême, les deux tendances tournent au pathologique. Tandis que l'extrême concentration engendre les idées fixes, la distraction démesurée produit la fuite des idées. Il faut mentionner aussi des troubles de civilisation comme le déficit de l'attention ou l'hyperactivité, étiquetés comme attention deficit syndrome (ADS) ou attention hyperactivity syndrome (ADHS) et expliqués entre autres par un manque de dopamine. Il n'est pas facile de décider à partir de quel point nous entrons sur le terrain d'une surmédicalisation, soutenue par l'industrie pharmaceutique et basée sur le monolinguisme d'une unified medicine.

Ensuite, nous pouvons mentionner le cas du blocage de l'attention. Les deux ailes de l'attention, respectivement constituées par l'événement d'être

16. Le concept de phénoménotechnique remonte à l'épistémologie de Gaston Bachelard; je l'utilise dans un sens plus large. Cf. Bruchlinien der Erfahrung, loc. cit., ch. VIII.

17. C'est Husserl qui parle d'un «Vorzimmer des Ich ». Cf. Analysen zur passiven Synthesis (Hua XI), Den Haag, M. Nijhoff, 1966, p. 166 (trad. fr. par B. Bégout et J. Kessler, De la synthèse passive. Logique transcendantale et constitutions originaires, Grenoble, Jérôme Millon, 1998, p. 231). 


\section{L'attention}

affecté par... et par l'événement de répondre à..., peuvent se déconnecter dans une mesure telle que l'attention en vienne à se briser. C'est au terme de ce processus que se trouvent les possibilités extrêmes comme celle de quelqu'un qui est frappé sans être capable de répondre ou, inversement, qui répond sans être atteint. D'une part, nous découvrons le phénomène de choc qui paralyse notre comportement d'une manière décrite par Descartes sous le titre d'étonnement ${ }^{18}$, ainsi que le phénomène de fascination qui fait que notre regard et notre désir sont captivés. D'autre part, nous rencontrons des stéréotypes, à savoir des formes pétrifiées de réponse, privées de toute inquiétude et de toute impulsion. Cette déviation pathologique se trouve clairement illustrée par Herman Melville sous la figure de Bartleby. Son histoire est celle d'un scribe qui tout à coup refuse son service, ne cessant plus de répéter la seule formule : «I would prefer not to ». En recourant aux stéréotypes purs on s'approche de l'état de mort, constitué par l'indifférence où tout est égal ; l'attention s'éteint comme un feu.

Finalement, il faut tenir en compte le traumatisme, qui peut être défini comme une irruption particulièrement grave. En général, le traumatisme est entendu comme un événement blessant et violent, bloquant toute réponse. Le patient est ce qui lui arrive. C'est un événement prématuré qui ne se manifeste qu'après coup et de façon indirecte, sous la forme des symptômes. Pour pénétrer l'armure servant à la protection de soi, Freud propose des méthodes spéciales comme l'attention flottante ou l'association libre, qui ressemblent à la remise en mouvement d'un mobile.

\section{Attention dirigée}

Comme nous avons déjà montré, l'attention prend son départ dans quelque chose qui nous arrive, dans la mesure où il suscite et attire notre attention: nous devenons attentifs lorsque nous remarquons quelque chose. Cependant, ce n'est pas une affaire privée. Vivre ensemble implique que $\mathrm{d}$ 'autres peuvent nous faire remarquer quelque chose ${ }^{19}$. Quelqu'un attire notre attention sur quelque chose qui est à regarder ou à écouter; il pourrait s'agir d'une fleur rare, d'une mélodie, d'une mauvaise mine, d'une tricherie politique, de gens affamés ou d'une victime dans la rue. Notre attention est donc plus ou moins dirigée par l'intervention des autres. Cette dimension sociale de l'attention a d'énormes implications; elle inclut l'enseignement, la rhétorique politique, des mesures thérapeutiques et la publicité économique. La question se pose donc de savoir de quelle manière et dans quelle mesure l'attention est imprégnée par le pouvoir ${ }^{20}$. Je ne choisis ici qu'un seul aspect, qui touche au cœur du problème. Ce qui est essentiel peut être illustré par la relation entre l'enseignant et le disciple. Lorsque les enseignants s'adressent

18. Voir le traité des Passions de l'âme, art. 73.

19. En allemand ont peut distinguer distinction entre Aufmerksamwerden et Aufmerksammachen.

20. Voir en détail ma Phänomenologie der Aufmerksamkeit, loc. cit., ch. IX. 
à certains disciples, en attirant leur attention sur quelque chose, ils ne font pas seulement ce qu'ils veulent, mais ils sont profondément engagés dans l'expérience qu'ils provoquent. C'est Freud qui présume que l'homme n'est pas maître dans sa propre maison; de façon semblable, on pourrait dire que l'enseignant n'est pas maître dans son école. Le succès de ce que l'enseignant fait dépend de la réponse de ceux à qui il apprend: il faut attendre que l'attention vienne. Comme le français le suggère, l'attention n'est pas seulement proche de l'intention, mais aussi proche de l'attente. En outre, celui qui enseigne est quelqu'un qui a été lui-même ou elle-même à l'école des autres. Éveiller l'attention de l'autre veut dire aussi être éveillé par d'autres. L'éducateur a lui-même besoin d'être éduqué, comme l'indique Karl Marx dans les Thèses sur Feuerbach. Si Socrate, le représentant principal d'un enseignement indirect, était banni de nos écoles et de nos universités, cellesci se changeraient en usines d'enseignement: l'attention dirigée dégénérait en attention fabriquée - ce qui, jusqu'à un certain point, est déjà la réalité que nous connaissons.

\section{Attention et respect}

L'attention n'est pas seulement quelque chose qu'on manipule et qu'on contrôle, en fin de compte c'est quelque chose que nous donnons ou refusons et que nous devons à autrui ${ }^{21}$. C'est pourquoi il $n^{\prime} y$ a pas $\mathrm{d}$ 'attention sans une impulsion éthique. À cet égard, la langue allemande est particulièrement éloquente : car l'attention en tant qu'Aufmerksamkeit y est étroitement liée à l'Achtung ou Achtsamkeit, c'est-à-dire au respect. Ainsi «faire attention" peut être rendu par acht geben. Mais pour vraiment tenir compte de ces suggestions, il faudrait en revenir à une éthique "d'en bas », à savoir à une éthique qui respecte les demandes singulières d'autrui avant se référer à des normes générales. En tout cas, l'attention s'avère plus qu'un accomplissement cognitif ; elle nous donne de grandes leçons pour un ethos des sens. Cet ethos de base émerge des actes de regard et d'écoute qui entrent en contraste avec la fermeture des yeux et des oreilles. En allemand, nous faisons une distinction entre hinsehen, hinhören, et wegsehen, weghören ou übersehen, überhören. Même Husserl parle d'une sorte de regard et d'écoute responsif (antwortendes Hinsehen und Hinhören) ${ }^{22}$. Dans ce contexte je me permets de citer encore une fois la littérature. Dans son poème Musée des Beaux-Arts, évoquant une réminiscence à Bruxelles, W.H. Auden écrit :

21. Le langage de l'attention produit des idiomes divergents. Des expressions comme Aufmerksamkeit schenken, to pay attention ou faire attention ne sont certainement pas complètement différents, mais les connotations diffèrent d'une langue à l'autre. La même remarque pourrait être valable en ce qui concerne les langues de l'Extrême-Orient.

22. Voir E. Husserl, Zur Phänomenologie der Intersubjektivität, Dritter Teil : 1929-1935 (Ниа XV), Den Haag, M. Nijhoff. 1973, p. 462 (trad. fr. par N. Depraz in Sur l'intersubjectivité, tome II, Paris, PUF, 2001, p. 354). 


\section{L'attention}

In Breughel's Icarus, for instance: how everything turns away

Quite leisurely from the disaster; the Ploughman may

Have heard the splash, the forsaken cry,

But for him it was not an important failure; the sun shone

As it had to on the white legs disappearing into the green

Water; and the expensive delicate ship that must have seen

Something amazing, a boy falling out of the sky,

had somewhere to get to and sailed calmly on ${ }^{23}$.

Comme la phénoménologie le montre et la poésie le réalise, l'éthique est profondément engagée dans l'œuvre des sens. Faire attention à ce qui nous frappe et nous touche s'avère éthiquement surdéterminé. L'attention vécue dépasse nos propres projets, et elle dépasse d'autant toutes les techniques et pratiques qui modèlent notre comportement. Si nous admettons une attention primaire, qui contribue à la genèse du monde et de la vie, il faut lui concéder certains traits d'une attention sauvage.

23. Trad. fr. par J. Lambert, in W.H. Auden, Poésies choisies, Paris, Gallimard, 1976, 2005 : «Dans l'Icare de Bruegel, par exemple : comme tout se détourne/De la catastrophe sans se presser; le laboureur a pu entendre/Le floc dans l'eau, le cri de désespoir,/Mais pour lui ce n'est pas un échec important, le soleil brillait/Comme il devait sur la blancheur des jambes disparaissant dans l'eau verte,/Et le coûteux, le délicat navire qui avait dû voir/Quelque chose de stupéfiant, un garçon précipité du ciel,/Avait quelque part où aller et poursuivait tranquillement sa course ». 